Chirurg 2014 $\cdot 85: 449$

DOI 10.1007/s00104-014-2748-4

Online publiziert: 30. März 2014

๑) Springer-Verlag Berlin Heidelberg 2014

O. Strobel · M.W. Büchler

Klinik für Allgemein-, Viszeral- und Transplantationschirurgie, Universität Heidelberg

\title{
Randomisierte kontrollierte Studie zur Pankreatoduodenektomie mit und ohne Drainage
}

137 Patienten, bei denen eine PD durchgeführt wurde $(n=68$ mit Drainage vs. $\mathrm{n}=69$ ohne Drainage). Die Behandlungsgruppen waren hinsichtlich demographischer Faktoren und wichtiger Risikofaktoren (Pankreastextur, Gangdurchmesser) vergleichbar. Als primäre Endpunkte wurden Morbidität und Mortalität bis 90 Tage postoperativ untersucht.

\section{Hintergrund und Fragestellung}

Die meisten gastrointestinalen Resektionen können ohne die Einlage prophylaktischer Drainagen sicher durchgeführt werden. Insbesondere angesichts der hohen Morbidität postoperativer Pankreasfisteln ist die Einlage prophylaktischer Drainagen nach Pankreasresektionen in den meisten Kliniken nach wie vor Routine. $\mathrm{Ob}$ die routinemäßige Drainageeinlage nach Pankreasresektionen sinnvoll ist, wird aktuell intensiv untersucht. Im Heft 06/2013 von Der Chirurg hatten wir eine beobachtende Studie kommentiert, die an 1122 Patienten den Einfluss der prophylaktischen Drainageeinlage untersuchte und zum Schluss kam, dass eine routinemäßige Drainageeinlage keinen Vorteil bringt [1]. Van Buren et al. verglichen nun in einer multizentrischen randomisierten kontrollierten Studie die routinemäßige Drainageeinlage mit dem routinemäßigen Verzicht auf Drainagen nach Pankreatoduodenektomie (PD) und distaler Pankreasresektion (DP).

\section{Methoden}

An 9 Zentren für Pankreaschirurgie in den USA (ca. 50 PD im Jahr) wurden zwischen 09/2011 bis 12/2012 357 Patienten gescreent und 282 Patienten mit Pankreasresektionen eingeschlossen, darunter

\section{Ergebnisse}

Die Studie wurde vom Data Safety Monitoring Board wegen einer überhöhten Mortalität in der Gruppe ohne Drainage bei Patienten mit PD abgebrochen. Für Patienten mit DP läuft die Studie weiter. Die Durchführung einer PD ohne Drainage war mit einer signifikant erhöhten Morbidität und deutlich erhöhter Mortalität verbunden. Die Rate klinisch relevanter Pankreasfisteln (20 vs. $12 \%)$, die Notwendigkeit interventioneller Drainagen (23 vs. 9\%), die Häufigkeit von Magenentleerungsstörungen (42 vs. 24\%), von intraabdominellen Abszessen (26 vs. 12\%) und anderen Flüssigkeitskollektionen ( $12 \%$ vs. $2 \%$ ) sowie die Häufigkeit schwerer Diarrhöen (17 vs. 3\%) waren alle in der Gruppe ohne Drainage signifikant erhöht. Insbesondere war jedoch die Mortalität bei PD ohne Drainage im Vergleich zu PD mit Drainage vierfach erhöht (12 vs. $3 \%, \mathrm{p}=0,097$ ), weshalb die Studie bei Patienten mit PD abgebrochen wurde.

\section{Diskussion}

Die Studie zeigt deutlich, dass ein Verzicht auf prophylaktische Drainagen bei allen Patienten mit PD nicht sicher ist. Diese Ergebnisse stehen nur scheinbar im Widerspruch zu den beobachtenden Studien, die nahelegen, dass die routinemäßige Einlage von Drainagen bei allen Patienten mit Pankreasresektionen ebenfalls nicht sinnvoll ist [1]. Unter Berücksichtigung beider Informationen ist wahrscheinlich ein risikoadaptiertes Vorgehen mit selektiver Drainageeinlage sinnvoll. Bei der Evaluation eines solchen selektiven Konzepts sind u. a. patientenbezogene Risikofaktoren (z. B. Pankreastextur), die chirurgische Ergebnisqualität (z. B. Fistelrate) sowie die Verfügbarkeit einer interventionellen Radiologie zu berücksichtigen. Zusätzlich ist zu beachten, dass prophylaktische Drainagen bei unauffälliger Förderqualität frühzeitig entfernt werden sollten, bevor sie etwa durch aufsteigende Infektionen Schaden anrichten.

\section{Korrespondenzadresse}

\section{PD Dr. O. Strobel}

Klinik für Allgemein-, Viszeralund Transplantationschirurgie, Universität Heidelberg, Im Neuenheimer Feld 110, 69120 Heidelberg

Oliver.Strobel@med.uni-heidelberg.de

Interessenkonflikt. O. Strobel und M.W. Büchler geben an, dass kein Interessenkonflikt besteht.

\section{Literatur}

1. Correa-Gallego $C$, Brennan MF, D'Angelica M et al (2013) Operative drainage following pancreatic resection: analysis of 1122 patients resected over 5 years at a single institution. Ann Surg 258:10511058 